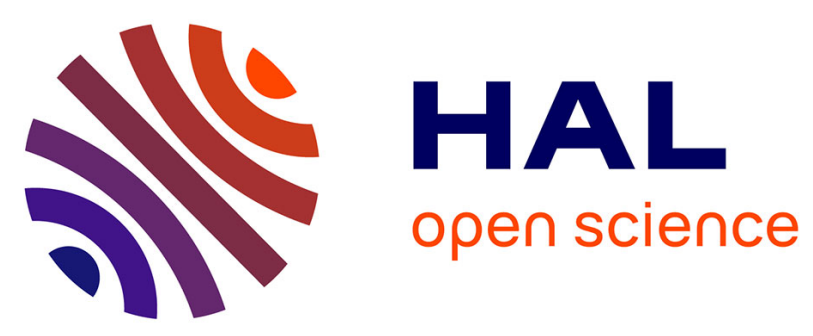

\title{
Les attendus implicites de la carrière: usages et mises en forme de la vie privée. Le cas d'une grande entreprise française
}

Cécile Guillaume, Sophie Pochic

\section{- To cite this version:}

Cécile Guillaume, Sophie Pochic. Les attendus implicites de la carrière: usages et mises en forme de la vie privée. Le cas d'une grande entreprise française. in Berrebi-Hoffmann I. (dir.). Politiques de l'intime - des utopies d'hier aux mondes du travail d'aujourd'hui, La Découverte, p. 145-167, 2009. halshs-01185014

\author{
HAL Id: halshs-01185014 \\ https://shs.hal.science/halshs-01185014
}

Submitted on 18 Aug 2015

HAL is a multi-disciplinary open access archive for the deposit and dissemination of scientific research documents, whether they are published or not. The documents may come from teaching and research institutions in France or abroad, or from public or private research centers.
L'archive ouverte pluridisciplinaire HAL, est destinée au dépôt et à la diffusion de documents scientifiques de niveau recherche, publiés ou non, émanant des établissements d'enseignement et de recherche français ou étrangers, des laboratoires publics ou privés. 
In BERREBI-HofFMANN Isabelle, 2009, Politiques de l'intime - des utopies d'hier aux mondes du travail d'aujourd'hui, La Découverte, «Recherches », p.145-167.

\title{
Les attendus implicites de la carrière : usages et mises en forme de la vie privée. Le cas d'une grande entreprise française.
}

\author{
Cécile Guillaume (CLERSE, Lille 1) et Sophie Pochic (CMH, CNRS)
}

Depuis quelques années, plusieurs enquêtes soulignent une évolution sensible des représentations de certains hommes, plutôt dans les jeunes générations et dans les classes moyennes, qui disent vouloir se distancier du modèle traditionnel du «chef de famille » et se (re)présentent comme le "compagnon » aidant d'une femme active dans une vision plus égalitaire de l'éducation des enfants. Ces jeunes hommes diplômés voudraient passer davantage de temps auprès de leurs enfants et aspirent à un équilibre entre vie professionnelle, vie familiale et vie sociale (Godechot, Lurol, Méda, 1999 ; Guichard-Claudic, 2005). Cette évolution des discours sur la paternité est sans doute liée à l'importance subjective accordée à l'enfant et à l'évolution de son statut dans la société (Gavarini, 2004), mais également aux transformations du marché du travail et notamment à la féminisation croissante du salariat (Maruani, 2003). Ces jeunes hommes ont ainsi de fortes probabilités d'être en couple avec une femme diplômée active susceptible de faire valoir une meilleure répartition des tâches parentales. Davantage contraints du côté de leur configuration conjugale et de la rhétorique légitime dans leur milieu social sur l'égalité entre les sexes, ces jeunes hommes adoptent donc souvent un discours égalitaire dont on peut néanmoins questionner la consistance.

Cette évolution partielle des discours cohabite en effet avec une très faible modification des pratiques, notamment sur le plan de la répartition du travail domestique (Dagenais, Devreux, 1998). Les enquêtes Emploi du temps de l'INSEE n'enregistrent pour l'instant qu'une très faible augmentation de la participation des hommes, qui respecte la division genrée - les pères s'impliquant plus dans les activités de sociabilité avec les enfants, tandis que les charges domestiques restent dévolues aux mères - (Barrère-Maurisson, Rivier, Marchand, 2000). Les hommes offrent ainsi une aide ponctuelle (Ferrand, 1984) ou une participation d'appoint, alors que leurs conjointes conservent toujours la charge mentale de l'articulation des sphères de vie (Haicault, 1984), et délèguent à d'autres femmes moins qualifiées. Entre ce nouvel idéal du « compagnon » et sa mise en pratique, il demeure donc encore un décalage important que l'on peut interpréter du point de vue de la recomposition des formes de domination masculine (de Singly, 1993 ; Ferrand, 2004) et du maintien d'un certain conservatisme dans la définition des rôles sexués, du côté des hommes comme de certaines femmes (Coehen-Hurter, 2001 ; Pochic, 2000). Cela peut s'interpréter aussi au regard du renforcement des attendus implicites qui conditionnent la possibilité d'une carrière promotionnelle dans les entreprises.

Même convaincus, les salariés d'entreprise (hommes ou femmes) semblent en effet aujourd'hui confrontés à la difficulté de faire entendre ce discours sur l'équilibre travail/horstravail dans l'espace professionnel. Le contraste entre la période actuelle et les années 1990 est sur ce plan-là assez saisissant. A l'époque du débat sur la réduction du temps de travail puis de la mise en place des 35 heures, et de la massification du chômage, de nombreux travaux (Gorz, 1988; Perret, Roustang, 1993; Méda, 1995) ont pris position en faveur de l'invention d'une société moins centrée sur le travail et valorisant l'engagement des individus dans d'autres sphères de vie. Quelques années plus tard, le discours sur un meilleur équilibre de vie n'a pas disparu, et peut même être repris par des services Ressources Humaines de grandes entreprises pour attirer les jeunes cadres. Mais le contexte de sa réception n'est plus aussi favorable et il reste majoritairement porté par certaines catégories de salariés - les femmes (Guillaume, 2006) et les jeunes. Plus que jamais, conserver son emploi et surtout faire carrière au sein d'une entreprise fonctionne sur le mode de la compétition individuelle et 
In BERREBI-HofFMANN Isabelle, 2009, Politiques de l'intime - des utopies d'hier aux mondes du travail d'aujourd'hui, La Découverte, «Recherches », p.145-167.

du sur-investissement dans le travail, particulièrement dans la population des dirigeants et des managers (et plus largement des élites économiques et politiques).

En nous appuyant sur une enquête menée dans une grande entreprise industrielle française qui avait pourtant été une des plus en pointe dans l'invention de formules collectives de réduction du temps de travail, y compris pour les cadres, nous montrerons d'abord cette permanence de normes de carrière masculines fondées sur le dévouement et la subordination des contraintes privées aux besoins de l'entreprise. Modèle de carrière porté par les dirigeants hommes actuels et qui reste faiblement questionné par les jeunes hommes cadres à potentiel. Nous analyserons ensuite, dans un contexte de féminisation croissante de la catégorie des cadres, la diversité des stratégies de ces femmes cadres pour faire face aux injonctions de mobilité et de disponibilité, en fonction de leurs ressources (diplôme et origine sociale).

\section{Encadré 1 : Carrières objectives et subjectives, approches plurielles}

Fortement inspirées par la tradition interactionniste sur les carrières «organisationnelles » (Hugues, 1937; Glaser, 1968), nous avons privilégié comme méthodologie les récits de vie au sein d'une grande entreprise du secteur de l'énergie afin de contextualiser les parcours des salariés en lien avec l'évolution des activités et des organigrammes, la politique formelle de Ressources Humaines et les normes informelles de détection/sélection. Ces récits permettent d'articuler les différentes étapes de la carrière avec les différents épisodes de la vie personnelle et familiale (mise en couple, naissances, déménagements, sociabilités...). L'échantillon final comprend 70 cadres, interviewés en 2004 et 2005, avec une surreprésentation délibérée des femmes. Il s'agit précisément de 30 cadres dirigeants à différents niveaux de l'organisation (16 femmes et 14 hommes, entre 39 et 58 ans), de 30 jeunes cadres étiquetés 'à potentiel' par leurs hiérarchiques ( 20 femmes et 10 hommes, entre 23 et 44 ans) et de 10 cadres 'lambda', considérés par l'organisation comme 'sans potentiel' (6 femmes et 4 hommes, entre 35 et 59 ans).

Afin de situer le plafond et les murs de verre de ce marché du travail, nous avons complété cet échantillon par une analyse secondaire du fichier du personnel, synchronique en 2004 puis longitudinal en 2006. Cette dernière base de données permet de retracer le parcours professionnel des salariés sur vingt ans (1986-2006), principalement en terme de carrière salariale (salaire de base sans les primes ni les bonus) et en terme d'évolution hiérarchique (niveau de responsabilités du poste). Elle permet aussi de retracer la mobilité fonctionnelle (en terme de «métiers» spécifiques à l'entreprise), la mobilité au sein de l'organisation (directions) et la mobilité géographique (lieu de travail). Elle contient quelques éléments sur la vie privée, comme la situation familiale actuelle (situation maritale et activité du conjoint, nombre d'enfants à charge) et la date de naissance des enfants, mais ne permet pas d'analyser la biographie familiale en dynamique.

Nous avons enfin réalisé une enquête par questionnaire sur les opinions des salariés sur l'égalité des chances et de la diversité au sein d'une direction parisienne de cols blancs, plus féminisée et jeune que la moyenne en 2005. Cette enquête à la demande de la direction a rencontré un vif intérêt, puisqu'elle a obtenu un taux de réponse de $80 \%$. Cette recherche a enfin été restituée en interne lors de conférences auprès de plus de 200 cadres en 2005.

\section{Un parcours de carrière construit au « masculin-neutre »}

\section{De la mobilité imposée à la mobilité négociée?}

Une des principales caractéristiques de l'entreprise étudiée est de proposer jusqu'à récemment aux cadres un déroulement de carrière linéaire et progressif, de l'embauche jusqu'à la retraite. Recrutés au premier niveau d'encadrement dans la grille de classification des emplois, les jeunes cadres sont appelés à monter progressivement les marches par le jeu d'une politique de mobilité fonctionnelle et/ou géographique systématique. La gestion des flux et l'organisation des mouvements de personnel visent officiellement à pourvoir l'ensemble des postes d'encadrement à Paris et en province, à alimenter un «vivier» de cadres disponibles et à éviter ainsi la vacance de poste. L'autre fonction de cette politique de mobilité tient dans la construction d'un parcours d'excellence pour les jeunes cadres prometteurs (école 
In BerReBI-HofFMANN Isabelle, 2009, Politiques de l'intime - des utopies d'hier aux mondes du travail d'aujourd'hui, La Découverte, «Recherches », p.145-167.

d'ingénieurs de groupe 1 et surtout Polytechniciens).

Les itinéraires de carrière des dirigeants masculins actuels projetés sur des cartes géographiques selon le principe d'Odile Join-Lambert (2001) dessinent donc en majorité des parcours en spirale, adaptés aux rythmes scolaires et familiaux. Après avoir passé de nombreuses années en province ${ }^{1}$, avec parfois des allers-retours Paris-province, au rythme d'une mobilité géographique tous les trois ou quatre ans, les cadres dirigeants se voient généralement offrir un poste en région parisienne (au siège) au moment où leurs enfants adolescents entrent en classe préparatoire et intègrent ensuite une Grande Ecole. L'accès à une base de données longitudinale interne retraçant les parcours professionnels des salariés sur vingt ans (1986-2006) nous a permis de tester ces hypothèses concernant la norme de la mobilité géographique et fonctionnelle pour accéder aux postes à responsabilité. Nous avons ainsi comparé les mobilités des salariés âgés entre 45 et 54 ans, cette restriction sur une tranche d'âge visant à neutraliser l'effet de l'ancienneté sur les indicateurs de changement. Les cadres sont beaucoup plus mobiles géographiquement que les non-cadres, mais les dirigeants se démarquent par leurs carrières extrêmement mobiles. Seuls 5\% des hommes dirigeants et $12 \%$ des femmes dirigeantes ont fait leur carrière dans le même département. Ce n'est le cas que de $25 \%$ des cadres masculins et $32 \%$ des cadres féminins, alors que cette sédentarité est la norme pour les non-cadres ( $63 \%$ pour les hommes et $65 \%$ pour les femmes). Dans ce contexte, ces équipes sédentaires de subordonnés qui effectuent les activités au quotidien et possèdent le savoir local assurent le relais entre des cadres qui se succèdent. Mais surtout les dirigeants masculins ne sont que $34 \%$ à avoir changé moins de trois fois de département de travail, alors que c'est le cas de $64 \%$ des cadres masculins du même âge. En comparaison, peut-être pour compenser leur moindre mobilité géographique ou en raison de spécialisation sur des fonctions support (communication, $\mathrm{RH}$ ), les femmes dirigeantes ont d'ailleurs davantage circulé entre les directions que leurs confrères, puisqu'elles ne sont que $28 \%$ à avoir changé moins d'une fois de direction, alors que c'est le cas de $50 \%$ de leurs confrères.

Cette pratique de la mobilité fonctionnelle et géographique est donc largement partagée par les cadres, hommes et femmes, et c'est surtout l'absence de mobilité - sédentarité ou spécialisation - qui est un handicap pour l'accès aux postes de dirigeants ${ }^{2}$. Avoir changé au moins une fois de département de travail et avoir changé au moins quatre fois de métiers depuis 1986 augmente de façon significative les chances d'être cadre supérieur ou dirigeant pour les hommes de plus de 45 ans (cf. annexe, tableau 1). Du côté des femmes, jouer le jeu de la mobilité géographique et/ou fonctionnelle et/ou en termes de directions augmente significativement les probabilités d'accéder aux niveaux supérieurs de la hiérarchie, mais à un niveau toujours inférieur à leurs confrères. Chez les dirigeants actuels (majoritairement des hommes), rares sont ceux qui ont dérogé à la règle de la mobilité imposée. Quand ils l'ont fait, c'est en général en raison du refus de leur conjointe de quitter la région parisienne ou de partir à l'étranger, et/ou afin de préserver leur couple d'un risque de divorce. Du côté des hommes, ces "ratés » de la mobilité imposée sont rares, comme l'atteste une enquête sur la banque (Bertaux-Wiame, 2008). Ceux qui ont réussi à ne pas trop en pâtir ont su jouer de la protection d'un « mentor » influent ou de leur réseau d'école, car la promotion fonctionne sur un principe informel de cooptation par les responsables hiérarchiques (Guillaume, Pochic, 2007). Surtout, tous ont compensé leur manque de mobilité par une disponibilité extensive.

\footnotetext{
${ }^{1}$ ou plus rarement dans les DOM-TOM, voire exceptionnellement dans des filiales européennes.

${ }^{2}$ Cette règle de la mobilité imposée comme condition sine qua non de la carrière promotionnelle se retrouve dans de nombreuses grandes entreprises réparties sur tout le territoire national, et notamment la banque, ce qui pose des problèmes spécifiques aux femmes avec enfants (Grafmeyer, 1990 ; Bertaux-Wiame, 2006).
} 
Cette politique de mobilité imposée par la hiérarchie sans négociation possible par le salarié sur le lieu d'affectation rencontre aujourd'hui des résistances du côté des jeunes générations. L'analyse statistique des itinéraires de carrière des jeunes cadres «à potentiel » (étiquetés comme possibles futurs dirigeants, entre 35 et 45 ans) révèle ainsi l'émergence de parcours moins mobiles géographiquement et fonctionnellement. Ces parcours alternatifs suivent l'évolution de l'activité de l'entreprise (internationalisation, commercialisation) et sont adossés à la construction d'une expertise pointue dans les directions parisiennes de cols blancs. Sous la pression des femmes cadres en couple à double carrière, beaucoup plus présentes dans les jeunes générations ( $45 \%$ des cadres de moins de 35 ans sont des femmes), la politique de mobilité systématique s'est assouplie et l'entreprise cherche des modalités pratiques d'organisation des mobilités professionnelles qui tiennent compte en partie des contraintes privées (missions courtes ou accompagnement de la recherche d'emploi du partenaire). Les jeunes hommes cadres à potentiel, davantage en couple à double carrière que leurs aînés (72\% d'entre eux sont dans un couple bi-actif, dont $64 \%$ avec une conjointe hors de l'entreprise, cf. annexe tableau 2), ont pu ainsi bénéficier de cette souplesse accordée aux nouvelles générations. Pour autant, l'organisation de la mobilité reste une nécessité pour l'entreprise, dans certaines branches, notamment dans les secteurs de cols bleus et en cas de réorganisations internes (appelées 'réorg' en interne) ${ }^{3}$. Elle entretient également des logiques de distinction dans un climat de compétition pour les postes les plus prisés ${ }^{4}$.

Dans le contexte de l'internationalisation du groupe, l'horizon de la mobilité géographique risque cependant de s'étendre à l'étranger, comme c'est déjà le cas pour certaines directions internationales ${ }^{5}$. Si le poste de «chef d'unité » sur le territoire national tend à perdre de son importance et de son prestige dans les réorganisations actuelles de l'entreprise, les fonctions de direction de filiale, surtout dans pays étrangers, deviennent les nouveaux postes stratégiques permettant de révéler les compétences managériales en contexte concurrentiel et parfois dans un climat social tendu (restructuration). Cette modification des périmètres du groupe contribue à re-créer une distinction entre les mobiles et les non-mobiles, comme dans d'autres multinationales (Forster, 1999). Les quelques cas de "grands experts» qui parviennent à restreindre leur exposition à la mobilité imposée, au prix souvent de nombreux déplacements à l'étranger, cache un renforcement probable des contraintes de mobilité géographique à l'international. On remarque en effet que si les conjointes de jeunes cadres masculins à potentiel refusent désormais de partir en province, particulièrement dans des petites villes ou des zones rurales, elles sont assez tentées par l'aventure internationale, au nom des atouts linguistiques et de l'ouverture d'esprit que cette expérience apportera aux enfants. Certaines sont donc prêtes à sacrifier leur carrière pour cette aventure exotique, ce qui s'observe encore rarement du côté des maris de femmes cadres à potentiel.

\section{La disponibilité extensive ou le modèle de la dévotion}

Le fait de «ne pas compter son temps» demeure toujours le deuxième critère-clé de détection/sélection essentiel pour être étiqueté «cadre à potentiel» et dès leurs premiers postes d'encadrement dans les directions de cols bleus, les jeunes cadres font l'apprentissage

\footnotetext{
${ }^{3}$ La fermeture ou transformation de services ou sites est moyen traditionnel pour faire évoluer les activités et métiers de l'entreprise avec un marché interne auparavant "fermé». Cette tendance semble s'accélérer récemment sous la pression de la concurrence, relayée par les conseils des consultants en organisation.

${ }^{4}$ Les cadres peu diplômés peuvent essayer de compenser par là leur handicap scolaire dans un système élitiste (Grafmeyer, 1990)

${ }^{5}$ Comme par exemple la direction chargée d'explorer et de gérer les gisements de gaz dans des pays éloignés, qui gère ses effectifs à l'échelle internationale et en incluant les filiales, où sont $90 \%$ de ses salariés. Cette internationalisation va sans doute s'accélérer avec sa fusion prochaine avec une autre multinationale.
} 
des contraintes de l'astreinte liée aux risques industriels des activités opérationnelles de transport et de distribution d'énergie. Dans la suite de leur carrière, le passage par des activités fonctionnelles hautement chronophages, comme le poste de "chef de cabinet ${ }^{6}$, vient encore renforcer l'idée que l'accès à une carrière promotionnelle est indissociable d'un engagement professionnel total. Cet accent mis sur la dévotion est caractéristique du fonctionnement des grandes entreprises, mais elle est ici encore renforcée par la culture du service public qui véhicule une vision idéalisée de la vocation et du dévouement (Saglio, 1999). Le bon manager loyal et dédié à son entreprise ne doit pas avoir d'autres engagements concurrents et ne doit pas laisser sa vie personnelle interférer avec ses obligations professionnelles (Palmer, 1996). Ce schéma de carrière hérité reste une référence commune, constamment validée par les dirigeants et gestionnaires de carrière en place, eux-mêmes souvent dégagés des contraintes familiales (enfants adultes).

Or le modèle de carrière managériale qui reste dominant dans l'entreprise s'appuie implicitement sur une division du travail asymétrique au sein du couple qui permet à l'un de se dédier pleinement à son investissement professionnel pendant que l'autre gère les « àcôtés » de sa carrière (Devreux, 1984 ; Nicole-Drancourt, 1989; Wajcman, 1996 ; Bonnet, Collet, Maurines, 2007). En raison des déménagements successifs (mais aussi du niveau de salaire croissant du conjoint), les femmes des dirigeants actuels ont rarement poursuivi une carrière, certaines ayant un emploi aux horaires limités, près de la moitié étant devenue inactive après la naissance des enfants (44\% d'elles, cf. annexe, tableau 2). Progressivement, leur configuration familiale est devenue de plus en plus traditionnelle, malgré parfois des aspirations égalitaires en début de couple. Soutien de la carrière de son mari, la « femme de cadre » gère les aspects familiaux et relationnels du projet de réussite sociale dont l'éducation des nombreux enfants, l'entretien d'un réseau de relations sociales et décharge son mari de la totalité des charges domestiques. Sa contribution varie en fonction de son niveau d'études et de ses qualifications (Pahl, Pahl, 1971; de Singly, Chaland, 2002) mais aussi de l'évolution de carrière du conjoint (Kanter, 1977). Cette configuration familiale traditionnelle (femme au foyer et famille nombreuse) est un facteur significatif au niveau statistique de l'accès aux postes de direction pour les hommes dans cette entreprise (cf. annexe, tableau 1), trait commun aux ingénieurs (Marry, 2002) et aux cadres en général (Pochic, 2005).

Si les cadres ont toujours eu des pratiques distinctes en matière de temps de travail (Laufer, 1998 ; Bouffartigue, 2001), les normes de disponibilité tendent aujourd'hui à se renforcer dans l'entreprise sous l'influence conjointe du contexte économique et politique (enjeux commerciaux, évolutions statutaires et réglementaires...) et des évolutions organisationnelles (restructurations, gains de productivité, baisse d'effectifs, organisation matricielle et en projet) dans un contexte d'internationalisation et de fusions-acquisitions incessantes (Pochic, Guillaume, 2009). De ce point de vue, les expériences collectives de passage à 32 heures, pourtant considérées comme innovantes au début des années 2000 notamment parce qu'elles ont également touché des cadres masculins ${ }^{7}$, semblent être en voie de raréfaction (à l'exception peut-être de la direction de la recherche). La disponibilité présentielle et le dévouement total à l'entreprise continuent d'alimenter des stratégies de distinction.

Si l'on constate des aspirations à plus d'égalité et de partage du côté des jeunes cadres, plus souvent en couple à double carrière que leurs prédécesseurs, on ne peut que souligner leurs difficultés à se mettre en porte-à-faux avec le modèle de manière frontale. Un questionnaire

\footnotetext{
${ }^{6}$ assistant personnel d'un patron de direction, sur le modèle de la haute fonction publique.

${ }^{7}$ Plusieurs enquêtes sur les cadres et les $35 \mathrm{~h}$ ont montré que si les cadres étaient plutôt favorables à cette réduction du temps de travail, les jours de RTT ont parfois généré une intensification du travail les autres jours travaillés (Bouffartigue, Bouteiller, 2002; Delteil, Génin, 2004). Les mères cadres avec de jeunes enfants semblent avoir le plus profité du desserrement des contraintes temporelles (Méda, Orain, 2002).
} 
passé auprès des salariés d'une direction parisienne à dominante commerciale et financière, révèle ainsi que les hommes cadres, et surtout les jeunes, sont majoritairement d'accord avec l'idée que les jeunes hommes veulent une vie plus équilibrée, passer plus de temps auprès de leurs enfants et surtout sont majoritairement en désaccord avec l'idée d'investissement prioritaire dans la vie professionnelle (cf. annexe, tableau 3). Faut-il y voir un effet de génération, d'âge, de cycle de vie ou un effet de position professionnelle (début de carrière versus poste à responsabilités) ? Certains jeunes pères adoptent en tout cas des stratégies « discrètes » de contournement qui les conduisent, par exemple, à arriver tard le matin pour avoir le temps d'emmener leurs enfants à l'école, ce qui n'est pas rare en région parisienne pour des cadres qui tentent d'éviter les heures de pointe et décalent leurs horaires dans la journée. D'autres refusent d'aller travailler avec des dirigeants connus pour leurs exigences en matière de disponibilité extensive. Surtout, de manière subtile, certains tentent d'alterner des postes très chronophages (management, chef de cabinet...) et d'autres plus tranquilles (experts) pour se caler avec leur cycle familial et être plus disponibles à certains moments (petite enfance) sans ternir leur réputation ${ }^{8}$. Prendre un peu de temps pour ses enfants (poser ses RTT le mercredi par exemple) peut d'ailleurs même être considéré comme une posture innovante de " nouveaux pères » dotés des bons atouts professionnels (diplôme prestigieux) dans certaines directions (dépendant du patron et du métier), alors que la même pratique renforce le stigmate de manque de disponibilité pour les mères de famille (Guillaume, Pochic, 2009).

Dans les couples à double carrière, quand l'un des deux doit modérer son investissement, c'est encore souvent la femme qui s'y résout. Cette décision est souvent « facilitée » par la difficulté des femmes à se fondre dans un modèle qui les pénalise. Forcément moins disponibles que leurs homologues hommes (ou les femmes sans enfant), "retardées » dans leur avancement de carrière par leur(s) grossesse(s) et moins mobiles (car leur conjoint est généralement lui aussi investi dans sa propre carrière de cadre), les mères de famille souffrent d'un handicap structurel qu'il leur est difficile de compenser.

\section{Les stratégies des femmes : contourner les normes sans les inverser}

Malgré tout, dans cette entreprise, quelques femmes ont réussi à passer le plafond de verre, puisqu'en 2007 près de $10 \%$ des cadres dirigeants sont des femmes, et un nombre significatif de jeunes femmes sont arrivées au seuil de ce plafond, puisque elles composent $35 \%$ du vivier des cadres étiquetés «à potentiel». Ces femmes cadres ne se sont pas toutes moulées dans le modèle masculin de la carrière «leader » et certaines ont essayé d'innover et de promouvoir d'autres règles du jeu, que ce soit dans leur couple ou dans leur propre service, par rapport à ces deux normes de mobilité et/ou de disponibilité. Révélatrices de la pluralité au sein du groupe des femmes diplômées ${ }^{9}$, ces stratégies sont cependant risquées, que ce soit du côté de la vie privée ou du côté de l'entreprise, et difficiles en pratique à tenir dans la durée.

\section{La carrière "leader 》 et son organisation matérielle}

Un petit nombre de femmes cadres ont accepté de se conformer à la norme de la mobilité imposée, le plus souvent quand elles étaient jeunes, célibataires et sans enfant. Pendant leurs

\footnotetext{
${ }^{8}$ Rester trop longtemps sur un poste de spécialiste était jusqu'à récemment risqué, les experts de la recherche ou en informatique étant considérés comme «hors-jeu» dans la compétition pour les postes d'encadrement et de direction.

${ }^{9}$ Pluralité au sein du groupe des femmes que l'on retrouve sur d'autres plans, la dimension symbolique des identités de genre (Lefeuvre, 2007) ou le rapport politique à l'ordre social sexué (Sénac, 2008).
} 
études, certaines d'entre elles ont souhaité avoir des expériences de mobilité nationale et internationale. Les "héritières" (fille de cadre dirigeant) ont parfois été socialisées à des déménagements successifs quand elles étaient enfant et considèrent la mobilité comme « normale » et neutre. Ces stratégies semblent être payantes, puisqu'on y retrouve les rares dirigeantes pionnières d'une cinquantaine d'années, ayant souvent aussi opté pour une famille peu nombreuse (un ou deux enfants), mais aussi des jeunes femmes à haut potentiel le plus souvent célibataires. La difficulté à gérer de front carrière et vie conjugale pour les femmes cadres se révèle statistiquement, puisque dans l'entreprise $25 \%$ des femmes dirigeantes vivent seules (célibataires ou divorcées), contre seulement $7 \%$ de leurs collègues hommes (cf. annexe, tableau 3$)^{10}$. Si certaine disent avoir délibérément « choisi» de rester seules pour se consacrer à leur carrière (Kaufmann, 1999), la plupart ont surtout rencontré des difficultés à trouver ou à conserver un compagnon acceptant leur mode de vie et leur réussite professionnelle. Les récits de carrière révèlent la difficulté d'organisation de cette carrière mobile au niveau familial, même dans les (rares) cas où le conjoint ne s'inscrit pas lui-même dans une carrière organisationnelle: activité libérale, expert, technicien, enseignant... Le suivisme du mari s'avère très compliqué dans la durée et bon nombre de tensions (voir de séparations) dans les couples où la carrière de la femme prédomine peuvent s'interpréter en partie à l'aune de cette hyper-conjugualité au féminin (Nicole-Drancourt, 1989). Le plus souvent, quand elles sont en couple ou seule avec de grands enfants, ces femmes adoptent une partie de la semaine le «célibat géographique» (Bertaux-Wiame, 2006). Quand les enfants sont petits, c'est en général avec elle qu'ils vivent la semaine, le mari faisant des déplacements ou ayant un pied-à-terre près de son travail. A l'inverse, quand les enfants sont grands, elles hésitent moins à partir plusieurs jours en semaine, laissant les enfants au domicile familial avec leur conjoint et/ou s'appuyant sur leur réseau familial (notamment quand elles sont divorcées).

\section{La mobilité de suivisme : les « tied movers»}

Les femmes cadres étant pour beaucoup en couple avec un homme cadre ${ }^{11}$, l'enquête a révélé de nombreux cas de mobilité de suivisme, ou «tied movers " (Bielby and Bielby, 1992), où les femmes ont accepté, parfois de manière répétée, de suivre leur conjoint en France et dans les DOM-TOM ou plus récemment à l'étranger. Contrairement aux stéréotypes sur «l'immobilité » supposée des femmes, nombre de femmes cadres en couple à double carrière ont précisément choisi cette grande entreprise pour son marché du travail national et donc les opportunités de mobilité possibles. Fruit d'une rencontre interne ou de stratégie de carrière " en couple ", on rencontre ici près de $10 \%$ de couples de cadres travaillant pour le même employeur. A l'initiative du salarié qui fait savoir la nécessité pour lui d'être mobile du fait de ses contraintes familiales, cette forme de mobilité à l'initiative du salarié déstabilise le modèle organisationnel de mobilité imposée qui prédomine ${ }^{12}$ et s'inscrit en porte-à-faux avec la temporalité normale de la carrière (trois ou quatre ans dans un même poste).

Si l'entreprise ne s'oppose pas à ces formes de mobilité à l'initiative du salarié en particulier quand elles se «justifient» du point de vue familial, elle ne les accompagne pas. Les salariés se débrouillent alors seuls pour trouver des points de chute, quitte à ne pas être très exigeants sur le contenu du poste. En début de carrière, dans cette situation, les femmes acceptent des

\footnotetext{
${ }^{10}$ En comparaison, la vie conjugale des cadres semble cependant plus stable que celles des salariés d'exécution, où $30 \%$ des hommes et même $33 \%$ des femmes vivent seules...

${ }^{11}$ En France, en 2002, les femmes cadres sont deux fois plus souvent que les hommes cadres en couple à double carrière avec un conjoint cadre, $48 \%$ contre $24 \%$ (source : enquête Emploi, Pochic, 2004).

${ }^{12}$ Les femmes demandent une mutation pour obtenir un lieu plutôt qu'un nouveau poste (Grafmeyer, 1990).
} 
In BERREBI-HofFMANN Isabelle, 2009, Politiques de l'intime - des utopies d'hier aux mondes du travail d'aujourd'hui, La Découverte, « Recherches », p.145-167.

mobilités horizontales sans promotion et parfois restent sur des "missions » en attendant qu'un poste se libère, en reconnaissant la chance qu'elles ont d'avoir une sécurité de l'emploi sur ce marché interne. En milieu de carrière, compte tenu de la restriction des espaces d'opportunité possibles, les femmes cadres peuvent même accepter un déclassement professionnel. Dans tous les cas, mobilité de suivisme rime avec retard de carrière et est fréquemment interprétée par la hiérarchie comme une "orientation familiale » qui peut être discriminante pour être détectée à potentiel. Rares sont donc les femmes dirigeantes qui ont adopté ce type de stratégie.

\section{La petite mobilité de " compromis » : la mobilité régionale sur un bassin d'emploi}

Qu'elle soit choisie ou subie, la mobilité géographique représente un risque pour la carrière (dans le cas du suivisme) et pour l'équilibre familial (dans le cas de la carrière leader). Nombreuses sont donc les femmes qui choisissent d'organiser leur carrière sur un bassin d'emploi donné, notamment la région parisienne, et de manière répétée, refusent des propositions de poste en province et parfois à l'étranger. Incarnant le modèle des " tiedstayers » (Bielby and Bielby, 1992), ce compromis implique de mettre en place des stratégies défensives à l'égard leur conjoint (Nicole-Drancourt, 1989), devant lui aussi faire valoir son immobilité, au prix parfois d'une carrière moins promotionnelle. En revanche, elles acceptent des affectations en grande banlieue, avec des temps de transport conséquents, des déplacements professionnels en France ou à l'étranger et des contenus de fonction souvent « obscurs » et a priori peu attrayants. L'avantage du marché de l'emploi parisien est de pouvoir offrir une alternance possible, bien que limitée, entre postes fonctionnels et postes opérationnels. Les directions distribution et transport offrent quelques opportunités d'acquérir une expérience managériale en île de France, même si seulement treize centres de distribution sont localisés en région parisienne (sur cent au total).

Ces stratégies de mobilité régionale semblent être tolérées "à la marge » par le système, comme des exceptions à la règle (dans lesquels on retrouve aussi les parachutés des Grands Corps directement au niveau des sphères dirigeantes, jusqu'à récemment surtout hommes). Les rares dirigeantes sur ce modèle sont en général en couple avec un autre cadre dirigeant et ont fait valoir leur non-mobilité dès le début de leur carrière, arguant le plus souvent de contraintes familiales. Elles ont compensé cet «handicap» par une intense mobilité fonctionnelle et une grande disponibilité, et bénéficiaient de la protection de leur diplôme prestigieux (grandes écoles, ingénieurs ou commerce). Cet ancrage local et leur niveau de revenu ont d'ailleurs facilité l'organisation de la vie familiale, par la sous-traitance des tâches domestiques et éducatives à un réseau stable de professionnelles (nounou, babysitter...) et parfois aux grands-parents.

Du côté de la norme de la disponibilité, on retrouve également cette diversité au sein du groupe des femmes cadres. Certaines tentent de se conformer au modèle dominant ou au contraire s'inscrivent en porte-à-faux avec ces conventions construites au masculin, d'autres cherchent des voies alternatives leur permettant de se préserver un espace de carrière.

\section{Se mouler dans le modèle de la disponibilité extensive}

Une première stratégie consiste en une mise en conformité avec le modèle temporel de la carrière, liant disponibilité professionnelle et rythme de progression (accéder au seuil des postes de direction avant 40 ans). Pour les jeunes femmes, cela suppose un report des projets 
de maternité jusqu'à l'atteinte d'un certain niveau de carrière et de réputation qui leur permettra de « rebondir » dans une seconde partie de carrière. Très répandu parmi les femmes célibataires ou sans enfant, mais également chez certaines femmes dirigeantes qui ont dû trouver les moyens de se conformer à cette norme du sur-investissement tout au long de leur carrière (notamment du fait de leur rareté dans l'entreprise), cette stratégie suppose une forte délégation du travail domestique à d'autres femmes, même si la charge mentale et l'organisation de cette sous-traitance reste l'apanage des femmes.

Chez les femmes pionnières, le soutien du conjoint (certaines d'entre elles ont connu une carrière plus promotionnelle que celle de leur mari), mais plus encore des grands-parents s'est avéré crucial dans la prise en charge des enfants. Pour les couples à double carrière, l'organisation de cette disponibilité horaire repose sur l'existence de modes de garde collectifs (relativement développés en France par comparaison avec d'autres pays européens), mais surtout sur une capacité financière suffisante pour rémunérer l'ensemble des salariés qui contribuent à la bonne marche de «l'entreprise domestique » (nounou, femme de ménage, baby-sitter, répétiteur...). Cette capacité à travailler " comme un homme » suppose également l'invisibilisation des absences pour motifs personnels, et notamment les absences pour enfants malades et les congés maternité, ainsi qu'une absence de culpabilisation par rapport au modèle de la « bonne mère ».

\section{« Concilier » travail et vie de famille}

Une deuxième stratégie consiste à l'opposé à revendiquer une « conciliation » vie privée-vie professionnelle qui accorde une place centrale à la sphère familiale, avec l'aspiration d'être une mère présente au quotidien. Plus répandue parmi les mères de famille avec de jeunes enfants, les cadres non détectés comme étant à potentiel et les cadres en position d'experts, ce modèle suppose un contrôle strict des horaires de travail et un recours fréquent au temps partiel scolaire (mercredi pour les enfants). Si les déterminants de ce "choix » du passage à temps partiel sont souvent difficiles à démêler (Guillaume, 2006), l'effet de plafonnement de carrière dû au diplôme de départ (en particulier pour les diplômés universitaires) comme l'orientation ou le maintien dans une position d'expert permet de saisir les conditions du choix comme du maintien du temps partiel dans la durée. Le nombre d'enfants, le rapport au modèle maternel et le type de transaction conjugale entrent également en ligne de compte. Ces femmes défendent souvent un nouveau rapport au temps qui n'implique pas un renoncement à la carrière (Laufer, 1998), mais parfois aussi un temps choisi moins « familialiste » ouvert à l'ensemble des salariés (Guillaume, 2001).

Cette réduction du temps de travail est très préjudiciable à la carrière quand elle intervient dans les premiers niveaux de carrière, au moment où les jeunes cadres doivent faire leurs preuves. Certaines femmes étiquetées « à potentiel », déjà reconnues pour leurs compétences, profitent des périodes où elles occupent un poste fonctionnel, notamment en direction centrale, pour faire « une pause » dans leur carrière ${ }^{13}$. Cette option est rarement envisagée pour des postes de management ${ }^{14}$, les rares femmes dans cette situation n'étant ni satisfaites de leur investissement professionnel, ni sereines dans l'organisation de leur vie privée. Rares sont celles qui se maintiennent dans un temps partiel dans la durée, ou alors au prix d'une « sortie durable de l'autoroute » et du vivier des cadres à potentiel. Comme le montre fort bien le calcul des probabilités d'accès à un poste de cadre dirigeant après 45 ans (cf. annexe,

\footnotetext{
${ }^{13}$ Même si elles acceptent souvent une forte porosité des temps, répondant au téléphone ou au mail, voire revenant pour une réunion importante pendant leurs jours non rémunérés, cette porosité travail/hors-travail augmentant avec l'avancée dans la carrière (Delteil, Génin, 2004).

${ }^{14}$ En l'absence de formule de « job-share » et surtout de soutien des hiérarchiques et des collègues.
} 
tableau 1), mais également d'autres études (Brockbank and Traves, 1996 ; Pochic, 2005 ; Guillaume, 2006), le recours au temps partiel annule toute chance d'accéder à un poste de dirigeant. L'entreprise peine à comprendre ce que certaines femmes considèrent comme une mise en parenthèse relative de leur carrière professionnelle et surtout à modifier ses critères d'âge (limite de 45 ans pour rentrer dans le vivier des potentiels). La rareté des compétences dans ce marché du travail relativement fermé peut cependant conduire certaines directions (notamment à la distribution) à relancer la carrière des femmes très diplômées à mi-parcours en leur offrant des postes d'encadrement, sous réserve d'un retour à temps plein. Suite à notre étude, le comité qui gère les parcours des cadres à potentiel a d'ailleurs mis en place un suivi individualisé systématique des femmes cadres à potentiel après 35 ans, afin d'éviter de perdre des ressources rares, considérés désormais comme «sans potentiel » par leur supérieur hiérarchique local en raison de leur moindre disponibilité à court terme.

\section{Inventer un modèle de "disponibilité flexible »}

Au-delà de ces deux modèles, une autre stratégie sans doute plus innovante émerge portée par une génération plus jeune de femmes dirigeantes ou à potentiel, parfois en position managériale et souvent mères de famille (parfois nombreuse) qui revendiquent une carrière professionnelle à l'égal des hommes et une vie familiale. Travaillant à temps plein, elles s'appuient sur une forte et coûteuse délégation des tâches domestiques et de garde d'enfant, mais s'arrangent pour consacrer la majeure partie de leur temps «libre » à l'éducation et au suivi scolaire de leurs enfants. Si elles conservent la charge mentale de l'organisation familiale, ce que l'une d'entre elles met en parallèle avec la position de " chef de projet », elles impliquent leur conjoint, lui-même cadre supérieur, dans la gestion a minima des contraintes familiales (le père a souvent la charge d'emmener les enfants à l'école le matin et de coordonner ses déplacements avec ceux de sa conjointe).

Sur le plan de l'organisation du travail, elles revendiquent une modération relative du temps de travail, essayant d'adapter leurs horaires à ceux de leurs enfants. Au prix d'une certaine intensification du travail (pas de pause déjeuner, peu de temps de sociabilités sur le lieu de travail, nombre de semaines de vacances limité) et d'une relative porosité des temps de travail et des temps privés (travail le soir après le coucher des enfants, usage extensif des moyens de communication à distance, mais également gestion de certaines contraintes familiales depuis le lieu de travail...), elles tentent de maintenir des temps familiaux « de qualité ». La plupart d'entre elles refusent de travailler le week-end, même si cette pratique semble très répandue chez les cadres dirigeants (mails envoyés le dimanche, travail le samedi matin...) et revendiquent une disponibilité flexible qui permet de maintenir une séparation de leurs « deux vies ». Cette gestion en double s'appuie sur une grande capacité d'organisation et une « culture du résultat » qui valorise davantage les compétences et les performances et minimise en retour la disponibilité présentielle comme critère d'évaluation du travail. Elle achoppe cependant parfois sur les limites physiques de ce co-investissement (Guillaume, 2006), certaines étant souvent proches du surmenage ( $"$ burn-out »).

\section{Une typologie des stratégies féminines de carrière}

Si l'on croise les trois types de stratégies à l'égard de la mobilité et de la disponibilité, on aboutit à une typologie de six modèles de carrière. L'acceptation de la mobilité imposée est toujours liée à l'adoption d'une disponibilité extensive au travail, traçant les contours du modèle (masculin) "spiraliste» de carrière promotionnelle hérité du passé et donc très répandu chez les femmes dirigeantes (plus de 45ans) et les jeunes femmes célibataires. Cette 
In BERREBI-HofFMANN Isabelle, 2009, Politiques de l'intime - des utopies d'hier aux mondes du travail d'aujourd'hui, La Découverte, «Recherches », p.145-167.

corrélation entre mobilité et disponibilité témoigne d'une forte orientation personnelle à l'égard de la carrière professionnelle et l'acceptation des coûts que cela implique sur la vie privée (« remise de soi » totale à l'entreprise). Si l'on s'intéresse aux femmes qui font valoir une mobilité réduite ou à l'échelle d'un bassin d'emploi (particulièrement les mères de familles avec de jeunes enfants), la typologie montre que trois choix s'offrent à elles. Elles peuvent soit compenser cette immobilité relative par une disponibilité extensive qui vise à masquer cette transgression partielle des normes (à l'image des rares hommes cadres sédentaires), soit défendre des normes de carrière alternatives minorant les contraintes de disponibilité présentielle au profit d'une culture du résultat, soit enfin assumer le choix d'une carrière subordonnée aux temporalités familiales (avoir une carrière " honorable », voire horizontale). Enfin, les femmes (et de plus en plus de jeunes hommes) en couple à double carrière doivent faire face à des contraintes de mobilité de suivisme qu'ils peuvent essayer de compenser tous les deux par une disponibilité extensive, ou alors qu'ils vont gérer de manière alternante (l'un des deux conjoints acceptant de minorer temporairement ses choix de carrière au profit de l'autre). Cette configuration peut enfin inciter les femmes à faire le « choix de la famille », au détriment de leurs propres perspectives de carrière, à l'image des générations antérieures, mais sans doute avec davantage de frustrations étant donné leur niveau de diplôme et de position professionnelle qu'elles avaient atteints (Bertaux-Wiame, 2008).

Typologies des stratégies féminines de carrière

\begin{tabular}{|c|c|c|c|}
\hline \begin{tabular}{l}
\multicolumn{1}{c}{1} \\
Disponibilité \\
Mobilité
\end{tabular} & Disponibilité extensive & Disponibilité flexible & $\begin{array}{l}\text { Conciliation vie privée- } \\
\text { professionnelle }\end{array}$ \\
\hline Mobilité imposée & $\begin{array}{l}\text { Conformité au modèle } \\
\text { « spiraliste » } \\
\text { (carrière leader ou célibataire) }\end{array}$ & $\mathrm{X}$ & $\mathrm{X}$ \\
\hline $\begin{array}{l}\text { Mobilité réduite/ } \\
\text { régionale }\end{array}$ & $\begin{array}{l}\text { Stratégie de compensation } \\
\text { (femmes dirigeantes et à très haut } \\
\text { potentiel) }\end{array}$ & $\begin{array}{l}\text { Modèle de carrière } \\
\text { alternatif } \\
\text { (femmes dirigeantes ou à } \\
\text { potentiel avec enfants) }\end{array}$ & $\begin{array}{l}\text { Modèle de carrière } \\
\text { «familiale » } \\
\text { (femmes expertes, parfois à } \\
\text { temps partiel) }\end{array}$ \\
\hline Mobilité de suivisme & $\begin{array}{l}\text { Couple à double carrière } \\
\text { (femmes en couple avec un cadre } \\
\text { supérieur/dirigeant) }\end{array}$ & $\begin{array}{l}\text { Modèle de la carrière } \\
\text { « alternante » } \\
\text { (femmes à potentiel en } \\
\text { couple avec un cadre et } \\
\text { des enfants) }\end{array}$ & $\begin{array}{l}\text { Modèle de carrière } \\
\text { « familiale » } \\
\text { (femmes expertes, parfois à } \\
\text { temps partiel) }\end{array}$ \\
\hline
\end{tabular}

Cette typologie permet de mesurer tout à la fois la force du modèle hérité et l'étroitesse de stratégies alternatives possibles. Pour une grande majorité de femmes, la seule stratégie possible est celle d'un "contrôle continu» (Monchatre, 1997) qui traduit une capacité d'anticipation fortement contrainte par le souhait de concilier projets professionnels et contraintes familiales (Guichard-Claudic, 2005). La mise en avant des contraintes familiales ou personnelles, du point de vue des critères de mobilité ou de disponibilité, accule les individus à tenter de masquer et/ou compenser cette transgression des normes par des stratégies de négociation sur les horaires de travail et les affectations souvent précaires dans la durée et toujours périlleuses du point de vue des perspectives de carrière et des équilibres familiaux. Pourtant, de plus en plus de femmes et d'homme à potentiel tentent de faire valoir d'autres modes d'organisation et d'évaluation du travail visant à la fois une plus grande efficacité du travail et la prise en compte (relative) des contraintes familiales et de la carrière en double. Cela dit, le caractère individuel de ces stratégies souligne la difficulté à porter un discours collectif, par défaut d'identification d'intérêts communs (et de lieux où les porter), 
In BERREBI-HofFMANN Isabelle, 2009, Politiques de l'intime - des utopies d'hier aux mondes du travail d'aujourd'hui, La Découverte, « Recherches », p.145-167.

mais aussi parce que ce registre d'action individuel est sans doute le seul qui soit disponible et légitime dans l'entreprise, particulièrement au sein d'une population de cadres très diplômés, eux-mêmes très en phase avec l'individualisation des modes de gestion... Bien qu'assez volontaristes (Laufer, Silvera, 2006) notamment en matière d'égalité salariale ${ }^{15}$, les deux accords égalité professionnelle signés par l'entreprise peinent, pour leur part, à aborder la question des normes de carrière qu'il s'agisse de la régulation des horaires de travail ou des contraintes de mobilité ${ }^{16}$. Comme le reflètent nombre d'accords (Guillaume, 2008), la négociation porte souvent sur des mesures spécifiques et individuelles de conciliation vie privée-vie professionnelle pensées pour les femmes et plus particulièrement les mères de famille, comme le temps partiel, qui contribuent en retour à renforcer les stéréotypes de sexe, sans déstabiliser les modèles de carrière dominants.

\section{Bibliographie}

Barrère-Maurisson Marie-Anne., Rivier S., Marchand Olivier (2000) « Temps de travail, temps parental. La charge parentale : un travail à mi-temps », Premières synthèses DARES, $\mathrm{n}^{\circ} 20-1$, avril

Bertaux-Wiame Isabelle (2006) «Conjugalité et mobilité professionnelle : le dilemme de l'égalité » Cahiers du Genre, n41, p.49-73.

Bertaux-Wiame Isabelle (2008), « Les comptes privés de la banque : les cadres et leur famille à l'épreuve de la mobilité », in Linhart D., Pourquoi travaillons-nous? Une approche subjective de la subjectivité au travail, Eres, p. 295-319.

Bonnet Estelle, Collet Beate, Maurines Béatrice (2006), «Carrière familiale et mobilité géographique professionnelle », Cahiers du Genre, n41, p.75-98.

Bielby William, Bielby Denise (1992) "I will follow him: family ties, gender-role beliefs, and reluctance to relocate for a better job”, The American Journal of Sociology, vol.97, n5, p. 1241-1267.

Bouffartigue Paul (2001), Les cadres. Fin d'une figure sociale, La Dispute.

Bouffartigue Paul, Bouteiller Jacques (2002), « Réduire le temps sans réduire la charge ? Les cadres et les $35 \mathrm{~h} »$, Travail et emploi, n 82 , p. 37-52.

Brockbank Anna, Traves Joanne (1996), «Career aspirations - women managers in retailing ", in Ledwith S., Colgan F. (eds), Women in organisations. Challenging gender Politics, Basingstoke, MacMillan, p. 78-98.

Coenen-Huther Jacqueline (2001), « Dominance et égalité dans les couples. Un réexamen de la théorie des ressources à la lumière de sous-cultures familiales », Cahiers du genre, $\mathrm{n}^{\circ} 30$, p. 179-204.

Dagenais Huguette, Devreux Anne-Marie (1998), « Les hommes, les rapports sociaux de sexe et le féminisme: des avancées sous le signe de l'ambiguïté », Nouvelles Questions Féministes, vol.11, n², p. 1-22.

\footnotetext{
${ }^{15}$ En 2004, dans un contexte particulier de relance des négociations sur l'égalité professionnelle (loi Génisson, 2001 ) et de mobilisation syndicale contre la privatisation de l'entreprise, un accord « volontariste » avait été signé comprenant une mesure-phare, une enveloppe de trois millions d'euros pour réduire l'écart salarial hommes/femmes sur trois ans. Cet accord a ensuite été renégocié en 2007.

${ }^{16}$ Plus aucune référence à l'obligation de mobilité géographique pour l'obtention d'une promotion n'est faite dans le nouvel accord.
} 
In Berrebi-HofFmann Isabelle, 2009, Politiques de l'intime - des utopies d'hier aux mondes du travail d'aujourd'hui, La Découverte, «Recherches », p.145-167.

Delteil Violaine, Genin Emilie (2004), «Les nouvelles frontières temporelles », In : Karvar A., Rouban L. Les cadres au travail, les nouvelles règles du jeu, La Découverte, p.39-56.

Devreux Anne-Marie (1984), «La parentalité dans le travail: rôles de sexe et rapports sociaux », In : Le sexe du travail, Presses Universitaires de Grenoble, p.118-126.

Ferrand Michèle (1984), «Paternité et vie professionnelle», In : Le sexe du travail, Presses Universitaires de Grenoble, p.127-139.

Ferrand M. (2004), « De la paternité, de la maternité et du féminisme. Entretien avec Michel Ferrand », Mouvements, $\mathrm{n}^{\circ} 31$, p. 45-55.

Flamand Nicolas (2002), Une anthropologie des managers, Paris, PUF.

Forster Nick (1999), « Another 'glass ceiling'? The experiences of women professionals and managers on international assignments », Gender, Work and Organization, vol.6, n², p. 7990 .

Gavarini Laurence (2004), «Le nouveau statut de l'enfant », Sciences Humaines, hors-série, $\mathrm{n}^{\circ} 45, \mathrm{p} .60-63$.

Glaser Barney (1968), The organizational career: a sourcebook for theory, Chicago, Aldine Publishing.

Grafmeyer Yves (1992), Les gens de la banque, PUF.

Guichard-Claudic Yvonne (2005), «Des projets d'avenirs féminins et masculins: entre convergence et reproduction des différences sexuées », Formation Emploi, n91, p.11-28.

Guillaume Cécile, «L'égalité professionnelle : entre préoccupation secondaire et tentation différentialiste », CFDT Cadres, n 432 , nov 2008, p.51-56.

Guillaume Cécile (2006) «Formes de socialisation professionnelles féminines au prisme du temps partiel choisi », in Erika Flahaut (sous la dir.), L'insertion professionnelle des femmes, entre contraintes et stratégies d'adaptation, PUR, p.157-167.

Guillaume Cécile (2001) «A l'épreuve du temps choisi », Cultures en mouvement, n³8.

Guillaume Cécile, Pochic Sophie (2009), «What would you accept to sacrifice? Access to top management and the work/life balance", Gender Work and Organisations, vol 16, ${ }^{\circ} 1$.

Guillaume Cécile, Pochic Sophie (2007), «La fabrication organisationnelle des dirigeants : un regard sur le plafond de verre », Travail, Genre et Société, n¹7, p. 79-104.

Gorz André (1988), Métamorphoses du travail. Quête de sens, Paris, Galilée.

Godechot Olivier, Lurol Martine, Méda Dominique (1999), « Des actifs à la recherche d'un nouvel équilibre entre travail et hors-travail », Premières synthèses DARES, ${ }^{\circ} 20.1$, avril.

Haicault Monique (1984), «La gestion ordinaire de la vie en deux », Sociologie du travail, $\mathrm{n}^{\circ} 3$, p. 268-277.

Hugues Everett C. (1937), «Institutional office and the person », The American Journal of Sociology, vol.43, n³, p. 404-413.

Join-Lambert Odile (2001), Le receveur des Postes, entre l'Etat et l'usager, Belin.

Kanter Moss Rosabeth (1977), Men and women in the corporation, NY, Basic Books.

Kauffman Jean-Claude (1999), La femme seule et le prince charmant. Enquête sur la vie en solo, Nathan.

Laufer Jacqueline (1998), «Les femmes cadres entre le pouvoir et le temps?», Revue 
In BerRebi-HofFmann Isabelle, 2009, Politiques de l'intime - des utopies d'hier aux mondes du travail d'aujourd'hui, La Découverte, «Recherches », p.145-167.

Française de Sciences Sociales, n³, p. 55-70.

Laufer J., Silvera R. (2006), «L'égalité des femmes et des hommes en entreprise. Des avancées dans la négociation ? », Revue de l'OFCE, p. 245-271.

Lefeuvre Nicky (2007), «Les processus de féminisation au travail: entre différenciation, assimilation et «dépassement du genre ». Entretien par Cécile Guillaume », Sociologies pratiques, $\mathrm{n}^{\circ} 14$, p.11-15.

Marry Catherine (2001), Les femmes ingénieurs, une révolution respectueuse?, Belin.

Maruani Margaret (2003), Travail et emploi des femmes, La Découverte.

Méda Dominique, Orain Renaud (2002), «Transformation du travail et du hors-travail : le jugement des salariés », Travail Emploi, n90, p. 23-38.

Méda Dominique (1995), Le travail, une valeur en voie de disparition, Aubier.

Montchatre Sylvie (1997), «Les déroulements de carrières en entreprise : variations sur le thème de l'anticipation. Le cas des techniciens et des cadres ", Sociologie du travail, ${ }^{\circ} 40(1)$, p. 21-40.

Nicole-Drancourt Chantal (1989), « Stratégies professionnelles et organisation des familles », Revue Française de Sociologie, vol.30, n², p. 57-80.

Pahl Jan M., Pahl Raymond E. (1971), Managers and their wife: a study of career and family relationship in the middle class, London, Allen Lane The Penguin Press.

Palmer Amanda M. (1996), " Something to declare - women in HM Customs and Excise », in Ledwith S., Colgan F. (ed), Women in organisations. Challenging gender Politics, Basingstoke, MacMillan, p. 125-151.

Perret Bernard, Roustang Guy (1993), L'économie contre la société, Paris, Esprit, Seuil.

Pochic Sophie, Guillaume Cécile (2009), «Les carrières des cadres au cœur des restructurations : la recomposition des effets de genre? », Sociologie du Travail, à paraître.

Pochic Sophie (2005), «Faire carrière : l'apport d'une approche en termes de genre », Formation - Emploi, n 91 , p. 75-94.

Pochic Sophie (2004), « Le chômage des cadres : un révélateur des tensions entre carrière et vie privée », Cahiers $d u$ Gdr Cadres, n5, p. 27-42.

Pochic Sophie (2000), «Comment retrouver sa place ? Chômage et vie familiale de cadres masculins », Travail, Genre et Société, n³, p.87-108.

Saglio Jean (1999), «Les fondements sociaux des hiérarchies salariales », Travail et Emploi nº 78 , p. 21-39.

Sénac-Slawinski Réjane (2008), L’ordre sexué. La perception des inégalités femmes-hommes, Paris, PUF.

Singly (de) François, Chaland Karine (2002), «Avoir le second rôle dans une équipe conjugale. Le cas des femmes de préfet et de sous-préfet », Revue Française de Sociologie, vol. $43, \mathrm{n}^{\circ} 1, \mathrm{p} .127-158$.

Singly (de) François (1993), «Les nouveaux habits de la domination masculine », Esprit, $\mathrm{n}^{\circ} 11, \mathrm{p} .54-64$.

Wajcman Judy (1996), "The domestic basis of the managerial career", Sociological Review, $\mathrm{n}^{\circ} 4, \mathrm{p} .609-629$. 
In Berrebi-Hoffmann Isabelle , 2009, Politiques de l'intime - des utopies d'hier aux mondes du travail d'aujourd'hui, La Découverte, « Recherches », p.145-167.

\section{Annexes}

Tableau 1 : Accès aux postes de cadres supérieurs et dirigeants après 45 ans

\begin{tabular}{|c|c|c|c|}
\hline$\%$ brut & Hommes & Femmes & Avantage masculin \\
\hline Moyenne & 14,5 & 6,1 & 2,4 \\
\hline \multicolumn{4}{|l|}{ DIPLÔME } \\
\hline Ecoles ingénieurs Groupe 1 & 52 & 25 & 2,1 \\
\hline Ecoles tertiaires Groupe 1 & 48 & 30 & 1,6 \\
\hline Ecoles ingénieurs Groupe 2 & 27 & 9 & 3,0 \\
\hline Autre diplôme du supérieur & 10,5 & 5 & 2,1 \\
\hline Bac ou moins & 0 & 0 & 0,0 \\
\hline \multicolumn{4}{|l|}{ MOBILITE GEOGRAPHIQUE } \\
\hline Aucune & 4 & 2 & 2,0 \\
\hline Plusieurs & 18 & 8 & 2,3 \\
\hline \multicolumn{4}{|l|}{ MOBILITE FONCTIONNELLE } \\
\hline Entre 0 et 3 changements de métiers & 7 & 2 & 3,5 \\
\hline $4 \mathrm{ou}+$ changements de métier ou plus & 16,5 & 7,5 & 2,2 \\
\hline \multicolumn{4}{|l|}{ MOBILITE ORGANISATIONNELLE } \\
\hline Aucune & 12 & 3 & 4,0 \\
\hline Plusieurs directions & 16 & 7,5 & 2,1 \\
\hline \multicolumn{4}{|l|}{ SITUATION FAMILIALE } \\
\hline Célibataire ou divorcé & 7 & 4,5 & 1,6 \\
\hline Marié conjoint actif dans l'entreprise & 12,5 & 5 & 2,5 \\
\hline Marié conjoint actif en dehors & 11,5 & 7,5 & 1,5 \\
\hline Marié conjoint(e) inactif (ve) & 22,5 & * & $*$ \\
\hline \multicolumn{4}{|l|}{ NOMBRE D'ENFANTS } \\
\hline 0 & 7 & 5,5 & 1,3 \\
\hline 1 à 2 enfants & 11 & 5 & 2,2 \\
\hline 3 enfants ou plus & 21 & 9 & 2,3 \\
\hline \multicolumn{4}{|l|}{ HORAIRES } \\
\hline Temps complet & 16 & 8 & 2,0 \\
\hline Temps partiel (collectif à 32h) & 0 & 0 & 0,0 \\
\hline Temps partiel (individuel) & 1 & 0 & 1 \\
\hline
\end{tabular}

Source : fichier du personnel 2006, avec données longitudinales 1986-2006, exploitation originale.

Effectifs : 3741 hommes (dont 543 dirigeants et supérieurs) et 800 femmes (dont 49 dirigeantes et supérieures).

Règle de lecture : en 2006, en moyenne 14,5\% des hommes cadres de plus de 45 ans sont sur un poste de cadre supérieur ou de dirigeant. S'ils possèdent un diplôme d'ingénieur d'une école prestigieuse (classée Groupe 1 par l'entreprise), ils sont $52 \%$ dans cette situation, contre $25 \%$ des femmes sorties de ces mêmes écoles. 
In Berrebi-Hoffmann Isabelle , 2009, Politiques de l'intime - des utopies d'hier aux mondes du travail d'aujourd'hui, La Découverte, « Recherches », p.145-167.

Tableau 2 : Des cadres à potentiel davantage en couple de bi-actifs que les cadres dirigeants

\begin{tabular}{|c|c|c|c|c|c|}
\hline En \% & $\begin{array}{l}\text { Célibataire } \\
\text { ou divorcé }\end{array}$ & $\begin{array}{c}\text { Marié conjoint } \\
\text { actif dans } \\
\text { l'entreprise }\end{array}$ & $\begin{array}{l}\text { Marié conjoint } \\
\text { actif en dehors }\end{array}$ & $\begin{array}{c}\text { Marié } \\
\text { conjoint(e) } \\
\text { inactif (ve) }\end{array}$ & Effectifs \\
\hline \multicolumn{6}{|l|}{$\begin{array}{l}\text { Dirigeants et } \\
\text { cadres supérieurs }\end{array}$} \\
\hline Hommes & $7 \%$ & $8 \%$ & $40 \%$ & $44 \%$ & 593 \\
\hline Femmes & $25 \%$ & $14 \%$ & $56 \%$ & $4 \%$ & 71 \\
\hline \multicolumn{6}{|l|}{$\begin{array}{l}\text { Cadres 'à } \\
\text { potentiel' }\end{array}$} \\
\hline Hommes & $16 \%$ & $8 \%$ & $64 \%$ & $12 \%$ & 226 \\
\hline Femmes & $31 \%$ & $14 \%$ & $53 \%$ & $2 \%$ & 125 \\
\hline \multicolumn{6}{|l|}{$\begin{array}{l}\text { Cadres } \\
\text { 'anonymes' }\end{array}$} \\
\hline Hommes & $18 \%$ & $9 \%$ & $51 \%$ & $22 \%$ & 5289 \\
\hline Femmes & $29 \%$ & $14 \%$ & $53 \%$ & $4 \%$ & 2040 \\
\hline \multicolumn{6}{|l|}{ Maîtrise } \\
\hline Hommes & $19 \%$ & $7 \%$ & $60 \%$ & $15 \%$ & 11355 \\
\hline Femmes & $32 \%$ & $17 \%$ & $46 \%$ & $5 \%$ & 5978 \\
\hline \multicolumn{6}{|l|}{ Exécution } \\
\hline Hommes & $30 \%$ & $3 \%$ & $50 \%$ & $17 \%$ & 5750 \\
\hline Femmes & $33 \%$ & $13 \%$ & $49 \%$ & $5 \%$ & 2267 \\
\hline \multicolumn{6}{|l|}{ Ensemble } \\
\hline Hommes & $21 \%$ & $6 \%$ & $55 \%$ & $18 \%$ & 23216 \\
\hline Femmes & $31 \%$ & $16 \%$ & $48 \%$ & $5 \%$ & 10481 \\
\hline Total & $24 \%$ & $9 \%$ & $53 \%$ & $14 \%$ & 33697 \\
\hline
\end{tabular}

Source : fichier du personnel 2006, exploitation originale. 
In Berrebi-Hoffmann Isabelle , 2009, Politiques de l'intime - des utopies d'hier aux mondes du travail d'aujourd'hui, La Découverte, « Recherches », p.145-167.

Tableau 3 : Des représentations sociales en évolution sur le partage des tâches

\begin{tabular}{|c|c|c|c|c|c|}
\hline $\begin{array}{c}\text { Je suis tout à fait d'accord / d'accord } \\
\text { avec les jugements suivants }\end{array}$ & Cadres & $\begin{array}{l}\text { lers } \\
\text { niveaux }\end{array}$ & Cadres & $\begin{array}{l}\grave{a} \\
\text { responsabilité }\end{array}$ & \\
\hline En \% & Hommes & Femmes & Hommes & Femmes & Total \\
\hline $\begin{array}{l}\text { Les jeunes salariés ont envie d'avoir une vie plus } \\
\text { équilibrée entre travail et hors travail. }\end{array}$ & 78 & 82 & 80 & 75 & 78 \\
\hline Les jeunes femmes veulent faire carrière. & 75 & 69 & 70 & 83 & 74 \\
\hline $\begin{array}{l}\text { Les jeunes hommes partagent davantage les tâches } \\
\text { domestiques. }\end{array}$ & 72 & 63 & 64 & 50 & 61 \\
\hline $\begin{array}{l}\text { Les jeunes hommes ont davantage envie de passer du } \\
\text { temps avec leurs enfants. }\end{array}$ & 59 & 57 & 60 & 33 & 54 \\
\hline $\begin{array}{l}\text { Le temps partiel (ou la réduction du temps de travail } \\
\text { à } 32 \mathrm{~h} \text { ) est mal vu pour un homme. }\end{array}$ & 55 & 48 & 63 & 75 & 54 \\
\hline $\begin{array}{l}\text { Les hommes s'investissent prioritairement dans leur } \\
\text { vie professionnelle. }\end{array}$ & 36 & 51 & 59 & 67 & 53 \\
\hline $\begin{array}{l}\text { Les femmes cherchent avant tout à équilibrer vie } \\
\text { professionnelle et vie privée. }\end{array}$ & 45 & 55 & 49 & 58 & 52 \\
\hline $\begin{array}{l}\text { Le temps partiel est un bon moyen pour concilier } \\
\text { travail-hors travail pour les femmes. }\end{array}$ & 44 & 61 & 54 & 25 & 53 \\
\hline Effectifs & 99 & 67 & 70 & 12 & 248 \\
\hline Taux de féminisation & & $40 \%$ & & $15 \%$ & $32 \%$ \\
\hline
\end{tabular}

Source : enquête par questionnaire dans une seule direction, novembre 2005.

NB : La distinction cadres de premier niveau / cadres à responsabilité repose sur la segmentation interne en terme de niveaux hiérarchiques et de niveau de salaire. Les 4 premiers niveaux ont été regroupés ensemble, tandis les trois niveaux supérieurs ont été regroupés avec les «cadres supérieurs » et les « cadres dirigeants ». Le taux de réponse de $80 \%$ permet d'avoir un échantillon représentatif en terme de taux de féminisation. 